\title{
Health apps targeting children with overweight-a protocol for a systematic review with meta-analysis and Trial Sequential Analysis of randomised clinical trials
}

Rajeeb Rashid ${ }^{1}$, Paolo Perego ${ }^{2}$, Laura Condon ${ }^{3}$, Janus Christian Jakobsen ${ }^{4,5}$, Jane Lindschou ${ }^{4}$, Christian Gluud ${ }^{4}$, Giuseppe Andreoni ${ }^{2}$ and Inge Lissau ${ }^{6^{*}}$

\begin{abstract}
Background: The prevalence of overweight is increasing worldwide in children. Multi-component interventions incorporating diet, physical activity, and behavioural change have been shown to reduce body mass index (BMI). Whilst many children have their own smartphone, the clinical effects of using smartphone applications (apps) for overweight are unknown. This systematic review aims to ascertain the effects of mHealth apps in children with overweight.

Methods: We will include randomised clinical trials irrespective of publication type, year, status, or language. Children between 0 and 18 years with overweight will be included. We will compare apps targeting overweight versus sham app, no app, or usual intervention. No distinction about operative system will be considered (i.e. Android, iOS, and Window Mobile will be included). The following databases will be searched: The Cochrane Library, Excerpta Medica database (Embase), PsycINFO, PubMed, IEEE Explore, Web of Science, CINAHL, and LILACS. Primary outcomes will be body weight, quality of life, and serious adverse event. Secondary outcomes will be selfefficacy, anxiety, depression, and adverse event not considered serious. Trial inclusion, data extraction, and bias risk assessment will be conducted independently by at least two authors. We will assess risk of bias through eight domains and control risks of random errors with Trial Sequential Analysis. The quality of the evidence will be assessed using Grading of Recommendations Assessment, Development and Evaluation Tool (GRADE).

Discussion: We will provide evidence of the beneficial and harmful effects of smartphone apps for children with overweight and highlight any gaps in the evidence in order to shape future potential interventions. By only including randomised clinical trials, we know that we bias our review towards benefits.
\end{abstract}

Systematic review registration: PROSPERO CRD42019120210

Keywords: Obesity, Overweight, Children, Adolescents, Smartphone app, Health app, mHealth app, Management, Intervention, Treatment

\footnotetext{
* Correspondence: info@ingelissau.dk

${ }^{6}$ Clinical Research Centre, University Hospital Copenhagen, Kettegard Alle 30,

2650 Hvidovre, Denmark

Full list of author information is available at the end of the article
}

(c) The Author(s). 2020 Open Access This article is distributed under the terms of the Creative Commons Attribution 4.0 International License (http://creativecommons.org/licenses/by/4.0/), which permits unrestricted use, distribution, and reproduction in any medium, provided you give appropriate credit to the original author(s) and the source, provide a link to the Creative Commons license, and indicate if changes were made. The Creative Commons Public Domain Dedication waiver (http://creativecommons.org/publicdomain/zero/1.0/) applies to the data made available in this article, unless otherwise stated. 


\section{Background}

The prevalence of overweight is increasing worldwide both among children and adults [1-3]. Despite significant resources being spent on reversing this trend, the rates of paediatric overweight have risen worldwide over the last few decades with an estimated 124 million obese children and adolescents $[4,5]$. This has also been associated with widening health inequality, as the prevalence of obese children is higher in areas of social deprivation [6]. Recent preliminary data by WHO European Childhood Obesity Surveillance Initiative have shown a decrease in childhood obesity prevalence over 10 years in Greece, Italy, Portugal, and Slovenia but acknowledges that changes are unequally distributed in all populations, again highlighting potential health inequality [7]. International Task Force of Obesity produced age- and sexspecific cut-off for the definition of overweight and obesity in children [8]. Throughout this paper, we will use the term overweight for all children with overweight including all levels of obesity.

Children with overweight will potentially have both short- and long-term comorbidities on cardiovascular disease, insulin resistance, type 2 diabetes, metabolic syndrome, and cancer (endometrial, breast, and colon). These result in a significant burden on the individual as well as health services across the world [5-9]. The severity of these comorbidities typically increases with the severity of overweight $[10,11]$ whilst mental health sequelae such as poor self-esteem, anxiety, and depression may result in bullying, discrimination, and longterm socioeconomic disadvantages [12-14].

\section{Mobile applications to support health (mHealth)}

During recent years, there has been an exponential global growth in Internet-connected devices such as smartphones for real-time communication, sharing of data, and running of multimedia software applications (apps). mHealth apps are software programs designed to support a healthy lifestyle and are among those most searched for and downloaded (Fig. 1) [15].

Children are a major consumer group for apps and subsequently present an opportunity to target the management of overweight in this population [16-19]. Apps thus represent a potentially effective medium for monitoring health parameters, interacting with individuals, and disseminating lifestyle interventions. However, paradoxically, apps can create an environment of social isolation, addiction, and anxiety through peer pressure and elevated sedentary activity through increased screen time [20-23].

Studies using mHealth have either used standalone apps or multi-component programmes which combine apps with direct sessions between the child and teacher/ clinician. Whilst many studies targeted healthy behaviours, only a few formally incorporated behavioural change theory in achieving these goals $[24,25]$. These apps were able to develop constructs based on self-determination or social cognitive theory to provide goal setting for nutrition, physical activity and screen time, tailored motivational messages, action planning, and reward systems. Such apps have the potential to support children with overweight to lose weight by supporting and strengthening their selfregulatory capacities $[24,26]$.

\section{Interventions in children with overweight}

Cochrane reviews from 2017 on interventions for overweight in younger children (6-11 years) and older children (12-17 years) highlighted the paucity of good quality trials on multi-component interventions incorporating diet, physical activity, and behaviour change. Few studies looked at changes in quality of life with

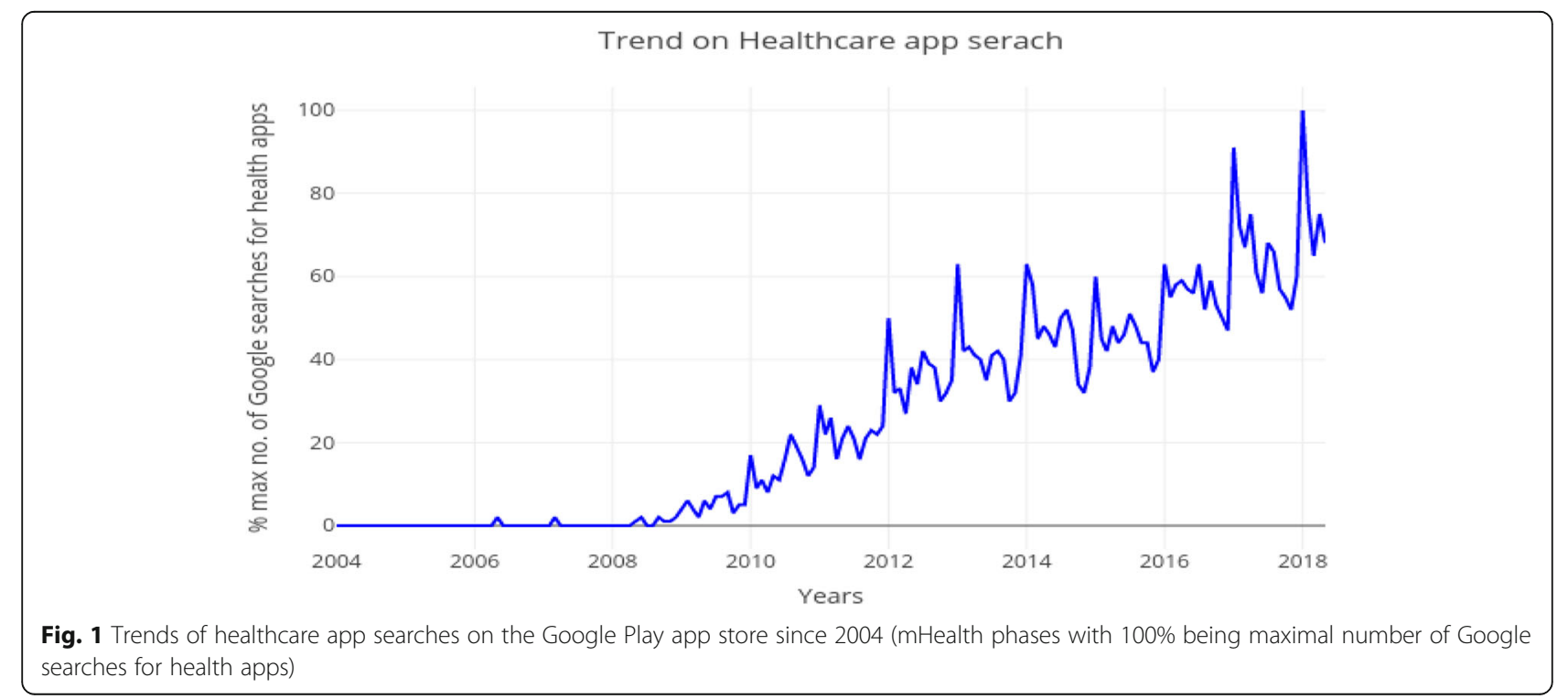


none showing any improvement in children post intervention and only a moderate improvement in older children, albeit in trials with a low quality of evidence [27, 28].

\section{Why is it important to do this review?}

The increasing availability of smartphones for children and families across all socio-economic groupings may enable the use of apps to deliver, promote, and sustain multi-component interventions which could lead to long-term improvements in health [19]. Apps could create a multi-domain and customisable approach by leveraging the personal interests and motivational dimensions to provide long-term efficacy. Only a few previous reviews have focused on smartphone apps interventions in children with overweight [29]. Whilst previous reviews have commented upon the significant risk of bias in many studies, there has not been a consistency in including control of bias or assessing the quality of evidence with the Grading of Recommendations Assessment, Development and Evaluation Tool (GRADE) [30-37]. Our protocol thus aims to outline an up to date systematic review focussed on health apps in children with overweight, searching multiple databases, analysing a range of anthropometric and psychosocial outcomes and utilising GRADE and TSA methodology to assess risk of bias and type I and II errors.

\section{Objective}

The objective of this review is to assess the benefits and harms of mHealth apps targeting children with overweight versus sham app, no app, or usual intervention in children with overweight.

In particular, we will analyse the results at three levels:

- General outcomes of intervention apps regardless of their specific strategy; no distinction about operational system will be considered (i.e. Android, iOS, and Window Mobile will be included).

- Specific analysis according to the type of structured intervention promoted by the app: on the increase of physical activity, on nutrition, on psychotherapy and education, or in an integrated approach.

- Specific analysis according to the age range of participants: pre-school age ( $0-5$ years), primary school age (younger children aged 6-11 years), secondary school age (older children aged 12 and below 18 years).

\section{Methods/design}

\section{Eligibility criteria}

Types of studies

Types of studies include randomised clinical trials irrespective of language, publication status, publication type, or publication year. Eligible studies which are not published in English will be translated using Google translate. By focusing on randomised clinical trials, we are aware that we focus on benefits and overlook harms. In case we find benefits of apps, systematic reviews on harms in observational studies need to be conducted.

\section{Types of participants}

Types of participants include all children who are overweight, up to 18 years of age. Children with associated co-morbidities, either physical or psychological secondary to overweight will be included.

\section{Types of intervention}

Types of intervention include all smartphone apps for intervention in children with overweight, independent of operating system and hardware platform. The control intervention can be a sham app, no intervention, or any current non-app intervention provided. There is no restriction for the duration of the intervention. Cointerventions are allowed if administered equally in the comparison groups.

\section{Grey literature}

There are many definitions of grey literature, but it is usually understood to mean literature that is not formally published in sources such as books or journal articles [38]. Conference abstracts and other grey literature have been shown to be sources of approximately $10 \%$ of the studies referenced in Cochrane reviews [39]. In a recently updated Cochrane methodology review, all five studies reviewed showed that published trials showed an overall greater treatment effect than grey literature trials [40]. Thus, failure to identify trials reported in conference proceedings and other grey literature might result in bias and affect the results of a systematic review.

\section{Outcomes}

One of the most commonly used outcomes to compare results from intervention studies is the BMI Z-score. Zscores are closely related to centiles and indicate the number of standard deviations the child's measurement lies above or below the mean or median reference value [41]. Similarly, the increasing use of equipment such as bioimpedance and dual energy X-ray absorptiometry (DEXA) have provided information on fat mass and muscle mass in kilogrammes which are also well understood by families and can provide a useful measure over time $[42,43]$. In addition, a serious adverse event will be defined as any untoward medical occurrence that results in disordered eating, significant or persistent morbidity, requires psychological or psychiatric treatment, hospitalisation or prolongation of existing hospitalisation. 
We will assess all outcomes at two time points:

- End of intervention-primary time point of interest

- Maximum follow-up

Primary outcomes

- Body weight measured in kilogrammes

- Quality of life as measured by any scale that has been validated for use in the target population [44]

- Proportion of participants with at least one serious adverse event [45]

Secondary outcomes

- BMI $z$-score

- Self-efficacy as measured by a scale validated for use in children

- Anxiety

- Depression

- Proportion of participants with at least one adverse event not considered serious

Exploratory outcomes

- Body fat (percentage) measured by bioimpedance or DEXA, good correlation having been shown between total body fat percentage and bioimpedance DEXA $(r=0.87, P<0.001)[42,43]$

- Muscle mass (kilogrammes) via bioimpedance or DEXA [42, 43]

- Individual serious and non-serious adverse events

\section{Search strategy}

We will search the following databases: Cochrane Library; MEDLINE; Excerpta Medica database (Embase); PsychINFO, IEEE Explore, Web of Science (SCI-Expended, SSCI, A\&HCI, CPCI-S, CPCI-SSH, ESCI, CCREXPANDED, IC), CINAHL, LILACS, OpenSIGLE, and Healthcare Management Information Consortium (HMIC).

In addition, we will search the following online resources: ClinicalTrials.gov (http://www.clinicaltrials.gov/), Google Scholar (https://scholar.google.com/), European Medicine Agency (https:// www.ema.europa.eu/ema/), United States Food and Drug Administration (www.fda. gov), Medicines and Healthcare Products Regulatory Agency (https://www.gov.uk/government/organisations/me dicines-and-healthcare-products-regulatory-agency), The World Health Organization (www.who.int/), Global Obesity Forum (previously International Association for the study of Obesity) (www.iaso.org), European Association for the study of Obesity (EASO) (easo.org), and ICTRP Search Portal.
Finally, keywords used in search strategies will be the following: Obesity, Overweight, Smartphone apps, Health apps, mHealth apps, Body Mass Index, Weight Gain, Weight Loss, and Hyperphagia (Additional file 1).

\section{Data collection process Selection of studies}

The review will follow the recommendations in the Cochrane Handbook for Systematic Reviews of Interventions and according to Keus and colleagues and Jakobsen and colleagues [46-49]. The analyses will be performed using Review Manager [50] and Trial Sequential Analysis programme [51]. Two authors (RR and $\mathrm{PP}$ ) will independently screen titles and abstracts. They will retrieve all relevant full-text studies/publications after which two authors will independently screen the full text in order to identify and record reasons for exclusion of the ineligible studies. We will resolve any disagreement through discussion. Trial selection will be displayed in an adapted flow diagram as per the Preferred Reporting Items for Systematic Reviews and Meta-Analyses (PRISMA) statement (Additional file 2).

\section{Data extraction and management}

Data extraction will be performed independently by at least two authors (PP and RR), who will both compare the extracted data. Disagreements will be resolved by a third author (GA or IL). We will assess duplicate publications and companion papers of a trial together to evaluate all available data simultaneously (maximise data extraction, correct bias assessment). Trial authors will be contacted by email to request any additional data which may not have been reported sufficiently or at all in the publication. Review Manager software will be used to extract data.

\section{Assessment of risk of bias in included studies}

The risk of bias of every included trial will be evaluated independently by at least two authors. In case of any disagreement, discrepancies will be discussed with a third author and resolved by consensus. The risk of bias will be assessed using the Cochrane's 'Risk of bias' assessment tool [52,53] and the Cochrane Effective Practice and Organisation of Care Group's guidance [54]. We will evaluate the methodology in respect of the following:

- Random sequence generation

- Allocation concealment

- Blinding of participants and treatment providers

- Blinding of outcome assessment

- Incomplete data outcome

- Selective outcome reporting

- Other risks of bias

- Overall risk of bias 
Classification of the trials will follow criteria defined in Additional file 3 [37, 55-60].

\section{Meta-analysis}

Both end scores and change-from-baseline scores will be used to analyse continuous outcomes. If both end scores and change-from-baseline scores are reported, then only end scores will be used. If only change-from-baseline scores are reported, these results together with end scores will be analysed in the same meta-analyses [61]. Exploratory outcomes will be analysed using change from baseline scores.

Data will be meta-analysed by RevMan 5 statistical software [50]. We will use STATA statistical software (STATA 2015) in case of zero event trials, where RevMan 5 zero event handling is insufficient $[62,63]$.

Intervention effects will be assessed by both randomeffects model meta-analyses and fixed-effect model meta-analyses [55, 64, 65], using the more conservative point estimate of the two. Three primary outcomes will be examined with $P \leq 0.025$ being statistically significant. An eight-step procedure will be used to assess if the thresholds for significance are crossed. Five secondary outcomes will be examined with $P \leq 0.017$ being statistically significant [48]. The results of the exploratory outcomes will be considered hypothesis generating only.

Analysis of all included studies will be compared to a sensitivity analysis of studies at low risk of bias. If the results are similar, primary conclusions will be based at the time point closest to 12 months on the overall analysis. If the results differ, primary conclusions will be based on studies with a low risk of bias.

A table describing the types of serious adverse events in each trial will be provided.

\section{Trial Sequential Analysis}

Traditional meta-analysis runs the risk of random errors due to sparse data and repetitive testing of accumulating data when updating reviews. Trial Sequential Analysis will thus be used to analyse the outcomes in order to calculate the required information size and control the risks of type I errors and type II errors [37, 56].

For continuous outcomes, Trial Sequential Analysis will use the observed SD, a mean difference of the observed $\mathrm{SD} / 2$, an alpha of $2.5 \%$ for the three primary outcomes, an alpha of $1.67 \%$ for the five secondary outcomes, and a beta of $10 \%$, with adjustment for observed diversity $[58,66]$. Mean differences (MDs) and the standardised mean difference will be expressed with 95\% confidence intervals (CI) for continuous outcomes, as well as the Trial Sequential Analysis-adjusted CIs for MDs.

For dichotomous outcomes, Trial Sequential Analysis will use the proportion of participants with an outcome in the control group, a relative risk reduction of $20 \%$, an alpha of $2.5 \%$ for primary outcomes, an alpha of $1.67 \%$ for secondary outcomes, and a beta of $10 \%$, with adjustment for observed diversity [58]. We will calculate risk ratios with 95\% CI for dichotomous outcomes, as well as Trial Sequential Analysis-adjusted CIs.

\section{Subgroup analyses}

Subgroup analysis when analysing the primary outcomes will be performed as follows:

- Trials at high risk of bias compared to trials at low risk of bias.

- Trials stratified according to experimental interventions.

- Trials stratified according to control interventions.

- Trials according to use of co-interventions.

- Complexity: trials with participants with no comorbidities compared to trials with participants preexisting co-morbidities.

- Trials in which the experimental intervention was evaluated by either the parents or the child after the treatment sessions had been delivered compared to trials in which the experimental intervention was not evaluated by either the parents or the child after the treatment sessions had been delivered.

We will use the formal test for subgroup interactions in Review Manager [50].

\section{Sensitivity analyses}

To assess the potential impact of bias, we will perform a sensitivity analysis to exclude trials at an overall 'high risk of bias'.

To assess the potential impact of the missing data for dichotomous outcomes, we will perform the following sensitivity analyses.

- 'Best-worst-case' scenario: assume that all participants lost to follow-up in the experimental group had no serious adverse events, including not developing any psychiatric disease such as an eating disorder.

- 'Worst-best-case' scenario: assume that all participants lost to follow-up in the experimental group, had a serious adverse event, for instance, developing a psychiatric disease such as an eating disorder.

Statistical heterogeneity will be assessed by visual inspection of the forest plots and $I^{2}$ statistic values [48]. Underlying reasons behind statistical heterogeneity in meta-analyses will be investigated by assessing trial characteristics. 


\section{Summary of findings table}

A summary of findings table using each of the prespecified primary outcomes will be presented using GRADE considerations for studies contributing data to the metaanalyses for the prespecified outcomes [48, 59, 60, 67-78]. Methods and recommendations described in Chapter 8 (Section 8.5) and Chapter 12 of the Cochrane Handbook for Systematic Reviews of Interventions will be followed using GRADEpro software [79].

\section{Discussion}

This review aims to provide evidence on the beneficial and harmful effects of smartphone apps as an intervention in children with overweight. Currently, there is no comprehensive systematic review of smartphone interventions in children with overweight to inform clinical practice. Previous systematic reviews in this population have considered the efficacy of mobile health technologies more broadly in the role of weight management [29], but none have provided comprehensive coverage of the benefits and harms of smartphone apps. Hence, this evidence will hopefully help children, their parents, and health professionals to make informed treatment decisions. This review will also highlight any gaps in the evidence base of such interventions which will help to shape the development and optimisation of future potential interventions.

This protocol has several strengths. The predefined methodology is based on the Cochrane Handbook for Systematic Reviews of Interventions and considering the risk of bias, Trial Sequential Analysis, and GRADE assessment $[61,64,75]$. We will assess both experimental and control interventions combined as well as individually, thereby being able to identify why interventions seem to work and under what conditions. This protocol, therefore, takes into account both the risks of systematic errors, the risk of random errors, and the risks of design errors [47].

The primary limitation of our protocol is that we are accepting interventions that have used all subtypes of smartphone apps. Hence, the different types of interventions with apps may have different effects compared with usual care, the statistical heterogeneity might be considerable and meta-analysis of all trials in one analysis might not be valid. A second limitation is the large number of subgroup analyses which increases the risk of a type I error. We have adjusted our thresholds for significance according to the number of primary outcomes, and the risk of type I errors and type II errors will be taken into account when interpreting the results of the review.

\section{Supplementary information}

Supplementary information accompanies this paper at https://doi.org/10. 1186/s13643-020-1269-0.

Additional file 1. Preliminary search strategy for MEDLINE (Ovid)
Additional file 2. Prisma-P+ checklist.

Additional file 3. Classification of randomised trials at low and at high risk of bias.

\section{Abbreviations}

Apps: Applications; BMI: Body mass index; Cis: Confidence intervals;

DEXA: Dual energy X-ray absorptiometry; GRADE: Grading of

Recommendations Assessment, Development, and Evaluation; MDs: Mean differences; mHealth: Mobile health; SD: Standard deviation; WHO: World Health Organization

\section{Acknowledgements}

We would like to thank Sarah Louise Klingenberg, Information Specialist at the Copenhagen Trial Unit, Centre for Clinical Intervention Research for her guidance and assistance on developing search methods keywords for search strategies.

\section{Authors' contributions}

$R R, I L, L C, P P, G A, J C J$ and $C G$ wrote the first draft of the protocol. RR, IL, PP, $G A, J C J, J$ and $C G$ revised the protocol. All authors read and approved the manuscript. IL is the guarantor for the review.

\section{Funding}

No funding has been received for this protocol.

\section{Availability of data and materials \\ Not applicable}

Ethics approval and consent to participate

Not applicable

Consent for publication

Not applicable

\section{Competing interests}

The authors declare they have no competing interests.

\section{Author details}

${ }^{1}$ Paediatric Unit, Department of Child Health, University of Edinburgh, St John's Hospital, Livingston EH54 6PP, UK. ${ }^{2}$ Department of Design, Politecnico di Milano, via Durando 38/a, 20158 Milan, Italy. ${ }^{3}$ School of Medicine, University of Nottingham, Medical School, Nottingham NG7 2RD, UK. ${ }^{4}$ Copenhagen Trial Unit, Centre for Clinical Intervention Research, Rigshospitalet, Copenhagen University Hospital, Blegdamsvej 9, 2100 Copenhagen $\varnothing$, Denmark. ${ }^{5}$ Department of Cardiology, Holbæk Hospital, Holbæk, Denmark. ${ }^{6}$ Clinical Research Centre, University Hospital Copenhagen, Kettegard Alle 30, 2650 Hvidovre, Denmark.

Received: 4 January 2019 Accepted: 2 January 2020

Published online: 11 February 2020

\section{References}

1. Wang Y, Lobstein T. Worldwide trends in childhood overweight and obesity. Int J Pediatr Obes. 2007/09/29. 2006;1(1):11-25.

2. Lobstein T. The prevention of obesity in children. Pediatr Endocrinol Rev. 2006/01/31. 2004;1 (Suppl 3):471-5.

3. de Onis M, Blossner M, Borghi E. Global prevalence and trends of overweight and obesity among preschool children. Am J Clin Nutr. 2010/ 09/24. 2010;92(5):1257-1264.

4. Jebb SA, Rennie KL, Cole TJ. Prevalence of overweight and obesity among young people in Great Britain. Public Heal Nutr. 2004/05/22. 2004;7(3):461-5.

5. WHO. Report of the commission on ending childhood obesity. Implementation Plan: Executive Summary [Internet]. Geneva, Switzerland: World Health Organisation; 2017. Available from: https://www.who.int/endchildhood-obesity/publications/echo-plan-executive-summary/en/

6. Olstad DL, Ancilotto R, Teychenne M, Minaker LM, Taber DR, Raine KD, et al. Can targeted policies reduce obesity and improve obesity-related behaviours in socioeconomically disadvantaged populations? A systematic review. Obes Rev. 2017/04/24. 2017; 
7. WHO. WHO Europe. COSI Factsheet. Childhood obesity surveillance initiative highlights 2015-17. Preliminary Data. 2018; Available from: http://www.euro. who.int/en/health-topics/disease-prevention/nutrition/publications/2018/ childhood-obesity-surveillance-initiative-cosi-factsheet.-highlights-2015-17-2018

8. Cole TJ, Bellizzi MC, Flegal KM, Dietz WH. Establishing a standard definition for child overweight and obesity worldwide: international survey. BMJ. 2000/05/08. 2000;320(7244):1240-3.

9. Kumar S, Kelly AS. Review of childhood obesity: from epidemiology, etiology, and comorbidities to clinical assessment and treatment. Mayo Clin Proc. 2017/01/10. 2017;92(2):251-265

10. Skinner AC, Perrin EM, Moss LA, Skelton JA. Cardiometabolic risks and severity of obesity in children and young adults. N Engl J Med. 2015/10/01. 2015;373(14):1307-17.

11. Kopelman P. Health risks associated with overweight and obesity. Obes Rev. 2007/02/24. 2007:8(Suppl 1):13-7.

12. Sawyer MG, Harchak T, Wake M, Lynch J. Four-year prospective study of BMI and mental health problems in young children. Pediatrics. 2011/09/21. 2011; 128(4):677-84

13. Griffiths $L J$, Wolke D, Page AS, Horwood JP. Obesity and bullying: different effects for boys and girls. Arch Dis Child.2005/09/22. 2006;91(2):121-5.

14. Gortmaker SL, Must A, Perrin JM, Sobol AM, Dietz WH. Social and economic consequences of overweight in adolescence and young adulthood. N Engl J Med. 1993/09/30. 1993;329(14):1008-12.

15. Andreoni G, Barbieri M, Colombo B. Emerging issues in healthcare: developing biomedical devices: Springer International Publishing; 2014.

16. Poushter J, Center PR. Smartphone Ownership and internet usage continues to climb in emerging economies [Internet]. Washington, USA: Pew Research Center; 2016. Available from: https://www.pewresearch.org/global/2016/ 02/22/smartphone-ownership-and-internet-usage-continues-to-climb-inemerging-economies/

17. Hwang Y, Jeong SH. Predictors of parental mediation regarding children's smartphone use. Cyberpsychol Behav Soc Netw. 2015/11/07. 2015;18(12): 737-43

18. Ofcom. Childrens and parents: media use and attitudes report [Internet]. UK; 2014. Available from: https://www.ofcom.org.uk/research-and-data/medialiteracy-research/childrens/children-and-parents-media-use-and-attitudesreport-2018

19. Mireku MO, Mueller W, Fleming C, Chang I, Dumontheil I, Thomas MSC et al. Total recall in the SCAMP cohort: validation of self-reported mobile phone use in the smartphone era. Env Res. 2017/11/03. 2018;161:1-8.

20. Davey S, Davey A. Assessment of smartphone addiction in Indian adolescents: a mixed method study by systematic-review and meta-analysis approach. Int J Prev Med. 2015/02/25. 2014;5(12):1500-11.

21. Kumar M. Impact of the evolution of smart phones in education technology and its application in technical and professional studies: Indian perspective. Int J Manag Inf Technol. 2011;3(3):39-49.

22. Derks D, ten Brummelhuis LL, Zecic D, Bakker AB. Switching on and off .... Does smartphone use obstruct the possibility to engage in recovery activities? Eur J Work Organ Psychol [Internet]. 2014;23(1):80-90. Available from: https://doi.org/10.1080/1359432X.2012.711013

23. Sarwar M, Soomro T. Impact of smartphone's on society. Vol. 98.2. European journal of scientific research; 2013. 216-226 p.

24. Anderson J, Kamphorst B. Ethics of e-coaching: Implications of employing pervasive computing to promote healthy and sustainable lifestyles. In: 2014 IEEE International Conference on Pervasive Computing and Communication Workshops (PERCOM WORKSHOPS). IEEE; 2014. p. 351-356.

25. Smith JJ, Morgan PJ, Plotnikoff RC, Dally KA, Salmon J, Okely AD, et al. Smart-phone obesity prevention trial for adolescent boys in low-income communities: the ATLAS RCT. Pediatrics. 2014/08/27. 2014;134(3):723-31.

26. Baroni I, Nalin M, Coti Zelati M, Oleari E, Sanna A. Designing motivational robot: how robots might motivate children to eat fruits and vegetables. In: The 23rd IEEE International Symposium on Robot and Human Interactive Communication. IEEE; 2014. p. 796-801.

27. Mead E, Brown T, Rees K, Azevedo LB, Whittaker V, Jones D, et al. Diet, physical activity and behavioural interventions for the treatment of overweight or obese children from the age of 6 to 11 years. Cochrane Database Syst Rev [Internet]. 2017 Jun 22;6:CD012651. Available from: https://doi.org/10.1002/14651858.CD012651

28. Al-Khudairy L, Loveman E, Colquitt JL, Mead E, Johnson RE, Fraser H, et al. Diet, physical activity and behavioural interventions for the treatment of overweight or obese adolescents aged 12 to 17 years. Cochrane Database
Syst Rev [Internet]. 2017/06/24. 2017 Jun 22;6:Cd012691. Available from: https://doi.org/10.1002/14651858.CD012691

29. Darling KE, Sato AF. Systematic review and meta-analysis examining the effectiveness of mobile health technologies in using self-monitoring for pediatric weight management. Child Obes. 2017/05/05. 2017;13(5):347-55.

30. Kjaergard LL, Villumsen J, Gluud C. Reported methodologic quality and discrepancies between large and small randomized trials in meta-analyses. Ann Intern Med. 2001/12/04. 2001;135(11):982-9.

31. Schunemann HJ, Wiercioch W, Brozek J, Etxeandia-Ikobaltzeta I, Mustafa RA, Manja V, et al. GRADE Evidence to Decision (EtD) frameworks for adoption, adaptation, and de novo development of trustworthy recommendations: GRADE-ADOLOPMENT. J Clin Epidemiol. 2016/10/08. 2017;81:101-10.

32. Savovic J, Jones $H$, Altman $D$, Harris $R$, Juni $P$, Pildal J, et al. Influence of reported study design characteristics on intervention effect estimates from randomised controlled trials: combined analysis of meta-epidemiological studies. Heal Technol Assess. 2012/09/20. 2012;16(35):1-82.

33. Schulz KF, Chalmers I, Hayes RJ, Altman DG. Empirical evidence of bias. Dimensions of methodological quality associated with estimates of treatment effects in controlled trials. Jama. 1995/02/01. 1995;273(5):408-12.

34. Wood L, Egger M, Gluud LL, Schulz KF, Juni P, Altman DG, et al. Empirical evidence of bias in treatment effect estimates in controlled trials with different interventions and outcomes: meta-epidemiological study. BMJ. 2008/03/05. 2008;336(7644):601-5.

35. Lundh A, Sismondo S, Lexchin J, Busuioc OA, Bero L. Industry sponsorship and research outcome. Cochrane Database Syst Rev. 2012;12.

36. Moher D, Pham B, Jones A, Cook DJ, Jadad AR, Moher M, et al. Does quality of reports of randomised trials affect estimates of intervention efficacy reported in meta-analyses? Lancet. 1998/09/24. 1998;352(9128):609-13.

37. Wetterslev J, Thorlund K, Brok J, Gluud C. Estimating required information size by quantifying diversity in random-effects model meta-analyses. BMC Med Res Methodol. 2010/01/01. 2009;9:86

38. Paez A. Gray literature: An important resource in systematic reviews. J Evid Based Med [Internet]. 2017;10(3):233-40. Available from:. https://doi.org/10. 1111/jebm.12266.

39. Mallett S, Clarke M. The typical cochrane review. Int J Technol Assess Health Care [Internet]. 2002;18(4):820-3 Available from: https://www.cambridge. org/core/product/identifier/S0266462302000624/type/journal_article.

40. Hopewell S, McDonald S, Clarke M, Egger M. Grey literature in metaanalyses of randomized trials of health care interventions. In: Hopewell S, editor. Cochrane Database of Methodology Reviews [Internet]. John Wiley \& Sons, Ltd: Chichester, UK; 2002. Available from:. https://doi.org/10.1002/ 14651858.MR000010.

41. Cole TJ R-CMF. Measurement and definition. In: Burniat W Lissau I, Poskitt EME, CT, editor. Child and adolescent obesity. Cambridge : Cambridge University Press; 2002. p. 3-27.

42. Pietrobelli A, Rubiano F, St-Onge MP, Heymsfield SB. New bioimpedance analysis system: improved phenotyping with whole-body analysis. Eur J Clin Nutr. 2004/05/13. 2004;58(11):1479-84.

43. Kabiri LS, Hernandez DC, Mitchell K. Reliability, validity, and diagnostic value of a pediatric bioelectrical impedance analysis scale. Child Obes. 2015/09/ 04. 2015:11(5):650-5.

44. Ahuja B, Klassen AF, Satz R, Malhotra N, Tsangaris E, Ventresca M, et al. A review of patient-reported outcomes for children and adolescents with obesity. Qual Life Res. 2013/06/27. 2014;23(3):759-70.

45. Proposal for a good clinical practice directive. Bull Med Ethics. 2001/10/20 1998;No. 135:6-11.

46. Davey J, Turner RM, Clarke MJ, Higgins JP. Characteristics of meta-analyses and their component studies in the Cochrane Database of Systematic Reviews: a cross-sectional, descriptive analysis. BMC Med Res Methodol. 2011/11/26. 2011:11:160

47. Keus F, Wetterslev J, Gluud C, Laarhoven CJ. Evidence at a glance: error matrix approach for overviewing available evidence. BMC Med Res Methodol [Internet]. 2010;10. Available from. https://doi.org/10.1186/1471-2288-10-90.

48. Jakobsen JC, Wetterslev J, Winkel P, Lange T, Gluud C. Thresholds for statistical and clinical significance in systematic reviews with meta-analytic methods. BMC Med Res Methodol. 2014/11/25. 2014;14:120.

49. Higgins JP GS. Cochrane Handbook for Systematic Reviews of Interventions Version 5.1.0 (updated March 2011) [Internet]. The Cochrane Collaboration. 2011. Available from: http://www.handbook.cochrane.org

50. Collaboration Cochrane. Review Manager. Nordic Cochrane Centre: Copenhagen; 2014. 
51. Kristian T, Engstrøm J, Wetterslev J, Brok J, Imberger G, Gluud C. User manual for trial sequential analysis (TSA). [Internet]. 2011. Available from: http://www.ctu.dk/tsa/files/tsa_manual.pdf

52. Sethi NJ, Safi S, Nielsen EE, Feinberg J, Gluud C, Jakobsen JC. The effects of rhythm control strategies versus rate control strategies for atrial fibrillation and atrial flutter: a protocol for a systematic review with meta-analysis and Trial Sequential Analysis. Syst Rev. 2017/03/08. 2017;6(1):47.

53. Higgins JP, Altman DG, Gotzsche PC, Juni P, Moher D, Oxman AD, et al. Cochrane Methods 2016 [Internet]. 2011/10/20. Chandler J, McKenzie J, Boutron I, Welch V, editors. Vol. 343, BMJ. 2016 Oct. Available from: https://doi.org/10.1002/14651858.CD201601

54. Mowatt G, Grimshaw JM, Davis DA, Mazmanian PE. Getting evidence into practice: the work of the Cochrane Effective Practice and Organization of care Group (EPOC). J Contin Educ Heal Prof. 2001/04/09. 2001;21(1):55-60.

55. DeMets DL. Methods for combining randomized clinical trials: strengths and limitations. Stat Med. 1987/04/01. 1987;6(3):341-50

56. Wetterslev J, Thorlund K, Brok J, Gluud C. Trial sequential analysis may establish when firm evidence is reached in cumulative meta-analysis. J Clin Epidemiol. 2007/12/18. 2008:61(1):64-75.

57. Thorlund K, Imberger G, Walsh M, Chu R, Gluud C, Wetterslev J, et al. The number of patients and events required to limit the risk of overestimation of intervention effects in meta-analysis - a simulation study. PLoS One [Internet]. 2011;6. Available from. https://doi.org/10.1371/journal.pone.0025491.

58. Castellini G, Nielsen EE, Gluud C. Comment on: "Cell therapy for heart disease: Trial sequential analyses of two cochrane reviews." Clin Pharmacol Ther. 2016/10/12. 2016;

59. Guyatt GH, Oxman AD, Vist GE, Kunz R, Falck-Ytter Y, Alonso-Coello P, et al. GRADE: an emerging consensus on rating quality of evidence and strength of recommendations. BMJ. 2008/04/26. 2008;336(7650):924-6.

60. Schunemann HJ, Best D, Vist G, Oxman AD. Letters, numbers, symbols and words: how to communicate grades of evidence and recommendations. Cmaj. 2003/10/01. 2003;169(7):677-80

61. Riley RD, Higgins JP, Deeks JJ. Interpretation of random effects metaanalyses. BMJ. 2011/02/12. 2011;342:d549.

62. StataCorp. StataCorp Statistical Software [Internet]. Release 14. College Station: StataCorp LP; 2014. Available from: http://www.stata.com

63. Sweeting MJ, Sutton AJ, Lambert PC. What to add to nothing? Use and avoidance of continuity corrections in meta-analysis of sparse data. Stat Med. 2004/04/30. 2004;23(9):1351-75.

64. DerSimonian R, Laird N. Meta-analysis in clinical trials. Control Clin Trials. 1986/09/01. 1986;7(3):177-88.

65. DerSimonian R, Laird N. Meta-analysis in clinical trials revisited. Contemp Clin Trials. 2015/09/08. 2015;45(Pt A):139-45.

66. Lloyd-Richardson EE, Jelalian E, Sato AF, Hart CN, Mehlenbeck R, Wing RR. Two-year follow-up of an adolescent behavioral weight control intervention. Pediatrics. 2012/07/04. 2012;130(2):281-8.

67. Guyatt GH, Oxman AD, Kunz R, Woodcock J, Brozek J, Helfand M, et al. GRADE guidelines: 7. Rating the quality of evidence--inconsistency. J Clin Epidemiol. 2011/08/02. 2011:64(12):1294-302.

68. Guyatt GH, Oxman AD, Kunz R, Woodcock J, Brozek J, Helfand M, et al. GRADE guidelines: 8. Rating the quality of evidence--indirectness. J Clin Epidemiol. 2011/08/02. 2011;64(12):1303-10.

69. Guyatt GH, Oxman AD, Sultan S, Glasziou P, Akl EA, Alonso-Coello P, et al. GRADE guidelines: 9. Rating up the quality of evidence. J Clin Epidemiol. 2011/08/02. 2011:64(12):1311-6.

70. Guyatt G, Oxman AD, Sultan S, Brozek J, Glasziou P, Alonso-Coello P, et al. GRADE guidelines: 11. Making an overall rating of confidence in effect estimates for a single outcome and for all outcomes. J Clin Epidemiol. 2012/ 05/01. 2013;66(2):151-7.

71. Guyatt GH, Oxman AD, Santesso N, Helfand M, Vist G, Kunz R, et al. GRADE guidelines: 12. Preparing summary of findings tables-binary outcomes. J Clin Epidemiol. 2012/05/23. 2013;66(2):158-72.

72. Guyatt GH, Oxman AD, Schunemann HJ, Tugwell P, Knottnerus A. GRADE guidelines: a new series of articles in the Journal of Clinical Epidemiology. J Clin Epidemiol. 2010/12/28. 2011;64(4):380-2

73. Garattini S, Jakobsen JC, Wetterslev J, Bertele V, Banzi R, Rath A, et al. Evidencebased clinical practice: overview of threats to the validity of evidence and how to minimise them. Eur J Intern Med. 2016/05/11. 2016;32:13-21.

74. Atkins D, Best D, Briss PA, Eccles M, Falck-Ytter Y, Flottorp S, et al. Grading quality of evidence and strength of recommendations. BMJ. 2004/06/19. 2004;328(7454):1490
75. Guyatt G, Oxman AD, Akl EA, Kunz R, Vist G, Brozek J, et al. GRADE guidelines: 1. Introduction-GRADE evidence profiles and summary of findings tables. J Clin Epidemiol. 2011/01/05. 2011;64(4):383-94.

76. Guyatt GH, Oxman AD, Kunz R, Atkins D, Brozek J, Vist G, et al. GRADE guidelines: 2. Framing the question and deciding on important outcomes. J Clin Epidemiol. 2011/01/05. 2011;64(4):395-400.

77. Guyatt GH, Oxman AD, Vist G, Kunz R, Brozek J, Alonso-Coello P, et al. GRADE guidelines: 4. Rating the quality of evidence--study limitations (risk of bias). J Clin Epidemiol. 2011/01/21. 2011;64(4):407-15.

78. Guyatt GH, Oxman AD, Montori V, Vist G, Kunz R, Brozek J, et al. GRADE guidelines: 5 . Rating the quality of evidence--publication bias. J Clin Epidemiol. 2011/08/02. 2011;64(12):1277-82

79. Collaboration TC. Green JPTH and S. Wiley-Blackwell: Cochrane Handbook for Systematic Reviews of Interventions. Cochrane Book Series; 2008.

\section{Publisher's Note}

Springer Nature remains neutral with regard to jurisdictional claims in published maps and institutional affiliations.
Ready to submit your research? Choose BMC and benefit from:

- fast, convenient online submission

- thorough peer review by experienced researchers in your field

- rapid publication on acceptance

- support for research data, including large and complex data types

- gold Open Access which fosters wider collaboration and increased citations

- maximum visibility for your research: over $100 \mathrm{M}$ website views per year

At $\mathrm{BMC}$, research is always in progress.

Learn more biomedcentral.com/submissions 